\title{
LA TESIS DE LOS LÍMITES COMPETENCIALES AL PODER DE REFORMA DE LA CONSTITUCIÓN DE COLOMBIA DE 1991: UNA MIRADA CRÍTICA DESDE LA DISCRECIONALIDAD JUDICIAL
}

\section{The Thesis of the Competence Limits to the Reform Power of the Colombian Constitution of 1991. A Critical Look from Judicial Discretion}

\author{
FRANKLIN MORENO MILLÁN' \\ Universidad Libre Seccional Cali \\ fmorenomillan@gmail.com
}

Cómo citar/Citation

Moreno Millán, F. (2016).

La tesis de los límites competenciales al poder de reforma de la Constitución de Colombia de 1991: una mirada crítica desde la discrecionalidad judicial. Anuario Iberoamericano de Justicia Constitucional, 20, 257-285. doi: http://dx.doi.org/10.18042/cepc/aijc.20.09

\section{Resumen}

La Corte Constitucional de Colombia, a lo largo de estos diez años construyó una dogmática de la teoría de los límites competenciales al poder de reforma que le

\footnotetext{
Abogado, magister en Derecho Público, candidato a doctor en derecho por la Universidad Rey Juan Carlos (España). Actualmente es profesor investigador de la Universidad Libre de Cali, y ha sido profesor invitado en diversas universidades. Autor de los libros La jurisprudencia constitucional como fuente del derecho (2002); la acción pública de inconstitucionalidad, efectos temporales de sus fallos (2007); la acción ciudadana de inconstitucionalidad (2013); y de artículos como "El concepto de paz en la Constitución Política de Colombia de 1991" (Revista de Derecho de la Universidad Católica del Norte, 2014) y "La refrendación de los Acuerdos de La Habana: del referendo constitucional a la asamblea nacional” (Revista de Derecho, 2015), entre otros.
} 
permitiera controlar el contenido de las reformas que se hacen a la Constitución Política de 1991. Sin embargo, aunque pareciera que dicha tesis —ella dice que es una teoría inacabada - se construyó sobre bases teóricas sólidas, lo cierto es que la misma puede ser objeto de fuertes críticas. Entre ellas, y la que se denuncia en el presente escrito, es que no tiene coherencia desde la teoría de la discreción judicial.

\section{Palabras clave}

Reforma constitucional; límites competenciales; Discrecionalidad judicial.

\section{Abstract}

The Constitutional Court of Colombia, over the last ten years built a dogmatic theory competency limits the power of reform that would allow control the content of the reforms made to the 1991 Constitution. However, although it seems that this thesis-she says she is an unfinished theory-was built on solid theoretical basis, the fact is that it can be subject to strong criticism. Among them, and that the complaint in the present paper, is that there is consistency from the theory of judicial discretion.

\section{Keywords}

Constitutional reform; jurisdictional limits; judicial discretion. 


\section{SUMARIO}

I. DEVENIR DOGMÁTICO DE LA TESIS DE LOS LÍMITES COMPETENCIALES AL PODER DE REFORMA DE LA CONSTITUCIÓN DE COLOMBIA DE 1991: 1. Primera etapa: negación de la tesis, años 1992 a 2003; 2. Segunda etapa: aceptación, 2003; 3. Tercera etapa: de maduración, 2004 a 2010; 4. La cuarta y actual etapa es de aplicación de la tesis: reafirmación. II. UNA MIRADA CRÍTICA DESDE LA DISCRECIONALIDAD JUDICIAL: 1. La discrecionalidad judicial; 2. De la discreción débil a discreción fuerte: control de vicios de competencia y la distinción entre reforma y sustitución. III. CONCLUSIONES. IV. BIBLIOGRAFÍA.

\section{DEVENIR DOGMÁTICO DE LA TESIS DE LOS LÍMITES COMPETENCIALES AL PODER DE REFORMA DE LA CONSTITUCIÓN DE COLOMBIA DE 1991}

La teoría de los límites competenciales se puede evaluar, en el caso colombiano, en cuatro etapas que condensan su devenir dogmático. A continuación una síntesis de cada una de esas etapas.

\section{PRIMERA ETAPA: NEGACIÓN DE LA TESIS, AÑOS 1992 A 2003}

Desde sus orígenes en la Constitución de Colombia de 1886, el control de constitucionalidad de los actos reformatorios de la Constitución Nacional (En adelante $\mathrm{CN}$ ) estuvo caracterizado por las siguientes reglas: era un control por vía de acción, rogado y circunscrito a los vicios de forma ${ }^{2}$.

2 La Acción de Inconstitucionalidad tuvo su origen en Colombia mediante Acto Legislativo 03 de 1910. El control concentrado tendría su origen precisamente en dicha reforma. El Tribunal Constitucional aparecería en el año 1920 con la obra de Hans Kelsen y las experiencias austriaca y checa. El modelo europeo, que tendría como antecedentes, según Ferreres Comella, la configuración del Tribunal Constitucional Austriaco, se daría a partir de las experiencias latinoamericanas. Sobre el origen de 
A diferencia de la CN de 1991, que permite el ejercicio de la acción de inconstitucionalidad (en adelante AI) contra sus actos reformatorios y la revisión automática y oficiosa en el caso de las leyes que convocan a referendo constitucional y a Asamblea Nacional Constituyente, el art. 154.4 de la Constitución de 1886 preveía que, a la Corte Suprema de Justicia (en adelante CSJ), le correspondía decidir sobre la exequibilidad de los proyectos de acto legislativo (en adelante AL) que hubieren sido objetados por el Gobierno como inconstitucionales, situación que se mantuvo con la reforma introducida por el AL 3 de 1910, que extendió a los ciudadanos la posibilidad de ejercer esta acción contra las leyes y los decretos.

Ya en el año 1991, con la adopción de una nueva Constitución y la creación de un tribunal especializado para la defensa de la Constitución, la situación no sufre mayores transformaciones. Si bien es cierto, se reconoce expresamente la posibilidad de que los ciudadanos inicien el control de constitucionalidad sobre los actos legislativos a través de la AI, también lo es que se restringe el control a los vicios de procedimiento ${ }^{3}$.

En igual sentido, el art. 379 expresa que la declaratoria de inconstitucionalidad de los reformas a la Constitución, en cualquiera de sus expresiones, «solo» procederá cuando se violen los requisitos establecidos en el título XIII constitucional.

Un examen desprevenido de este título, muestra únicamente requisitos de competencia y forma; por ejemplo, quiénes pueden presentar el proyecto de reforma, número de debates, mayorías requeridas y ciertos principios propios del procedimiento legislativo como el de consecutividad y el de identi$\mathrm{dad}^{4}$. Incluso, el hecho de limitar la procedencia de la $\mathrm{AI}$ al año siguiente a la promulgación de la reforma, como sucede con los vicios de forma, es clara muestra de las limitaciones que tiene la Corte Constitucional al ejercer su labor.

Conforme lo ha reconocido la jurisprudencia constitucional (SC-551, 2003), «el adverbio 'solo' no puede ser tomado en su sentido literal», en tanto otras normas de la Constitución, por ejemplo, los artículos 157.1 sobre publicación en la Gaceta del Congreso; 158 sobre unidad de materia; 160

la Acción de Inconstitucionalidad véase Moreno Millán (2013). Asimismo Esguerra Portocarrero (2004), Chinchilla Herrera (2010) y Favoreu (1994). Sobre las características del modelo europeo, Ferreres Comella (2011). También Cruz Villalón (1987).

3 Cf. Numeral $1^{\circ}$ del art. 241 de la Constitución Nacional de 1991.

4 Una explicación de estos principios en el procedimiento legislativo en Corte Constitucional. SC-040, 2010. 
en relación con el informe de ponencia y 169 sobre título del proyecto, son igualmente aplicables.

De la misma manera, la Ley Orgánica del Congreso 5/1992 (en adelante LOC), en lo pertinente y compatible (SC-222, 1997), pese a su naturaleza infraconstitucional, resulta aplicable al trámite de aprobación de las reformas a la Carta y, por ende, su desconocimiento puede derivar en una inconstitucionalidad de segundo grado (SC-387, 1997) ${ }^{5}$.

Desde sus primeros fallos, la Corte Constitucional aceptó que el análisis de las reformas a la Constitución estaba limitado a «aspectos formales y de trámite» (SC-222, 1997). Esta posición de autorestricción judicial, basada en el art. 241 superior, el cual preceptúa que la Corte ejercerá sus atribuciones «en los estrictos y precisos términos» de ese artículo, incluyó la negativa a revisar aspectos administrativos que escapan a un juicio de constitucionalidad y cuya verificación corresponde a otras autoridades. Esa verificación, dijo la Corte, se limita, a la «constatación de que se hayan cumplido a cabalidad todos los pasos del procedimiento agravado previsto para estas hipótesis en las normas superiores» (SC-387, 1997). Asimismo, expresó la Corte, está excluido del control constitucional «el contenido material del acto reformatorio» (SC-543, $1998)^{6}$.

En la SC-487, 2002, la Corte se inhibió de conocer de los cargos de fondo dirigidos contra una reforma constitucional. Así, luego de reseñar algunas acusaciones contra el contenido material del AL 1 de 2001, precisó que por tratarse de cargos referidos «al contenido material de las disposiciones acusadas», ella era incompetente y tenía que «inhibirse de hacer pronunciamiento de fondo".

Como característica especial de esta etapa, tenemos que el control por vicios de forma está limitado por el principio de congruencia que le impide a la Corte pronunciarse sobre aspectos no señalados por el accionante en la demanda.

En una primera posición, expresó el alto tribunal que a ella corresponde, «confrontar los disposiciones sometidas a control con la totalidad de los preceptos de la Constitución [...] [y] fundar una declaración de inconstitucionalidad en la violación de cualquiera norma constitucional, así ésta no hubiere sido invocada en el curso del proceso» (art. 22, Decreto 2067, 1991).

5 En igual sentido, Corte Constitucional. SC-543, 1998, SC-614, 2002 y SC-668, 2004.

6 En igual sentido Corte Constitucional, SC-487, 2002, donde se inhibe por tratarse de cargos referidos «al contenido material de las disposiciones acusadas». 
A esto agrega que si la Corte, «al examinar una demanda contra un acto legislativo, constata que éste adolece de vicios de procedimiento, es su deber examinarlos, incluso si éstos no fueron señalados por los actores» (SC-387, 1997).

Luego, el Tribunal Constitucional cambia de posición y expresa que «como el control constitucional de los actos legislativos no es de carácter oficioso, sino rogado (por demanda ciudadana), la Corporación en estos casos tan solo puede pronunciarse sobre los cargos formulados por los demandantes», con lo cual cierra este capítulo con una tesis que en lo sucesivo será sostenida sin variaciones $(\mathrm{SC}-543,1998)^{7}$.

\section{SEGUNDA ETAPA: ACEPTACIÓN, 2003}

La teoría de los límites competenciales al poder de reforma de la Constitución, a partir de la distinción entre reforma y sustitución, tendría acogida a partir del año de 2003, con la SC-551, 20038 previo, la Ley 796 de 2003, «por la cual se convoca un referendo y se somete a consideración del pueblo un proyecto de reforma constitucional».

En esta oportunidad, la Corte manifestó que las normas que sirven de parámetro para el control de constitucionalidad de los actos legislativos, son las del título XIII de la CN, según el perentorio mandato del art. 379 superior, pero que igualmente existen otras que surgen de los reenvíos de primer y segundo grado hechos por el art. 378. Entre las normas que constituyen reenvío de primer grado, están los arts. 115 sobre conformación del Gobierno, 155 sobre iniciativa popular, 241 y 242 sobre competencias de la Corte Constitucional, entre otros; el reenvío de segundo grado, está dado por las normas orgánicas que regulan la actividad legislativa (LOC) y la estatutaria sobre participación ciudadana (L. 134, 1994, en adelante LEMP).

La posibilidad hermenéutica para incluir en el proceso de control constitucional preceptos distintos a los establecidos en el título XIII, en contra de lo literalmente expuesto en el art. 379, surge de la ponderación entre los principios del efecto útil y de instrumentalidad de las formas?. Para el caso, el resultado de la ponderación, no es otro sino que la posibilidad de una declaratoria de in-

7 Posición reiterada por la Corte Constitucional en las SC-487, 2002, SC-614, 2002 y SC-1040, 2005.

8 Esta sentencia podría considerarse fundadora de línea. Una explicación de esta clasificación de las sentencias en López Medina (2009): 161.

9 Cf. Bernal Cuéllar y Montealegre Lynnet (1995: 284-285). Asimismo, Corte Constitucional. SC-737, 2001. 
constitucionalidad se circunscribe a aquellas irregularidades de una magnitud tal que constituyan vicios de procedimiento en su formación, entendiendo por tal la violación de los requisitos que la misma Carta fija para aprobar las reformas, con la inclusión de la LOC y la LEMP (SC-551, 2003).

A partir de tal consideración, la Corte concluye que la revisión de los actos reformatorios de la $\mathrm{CN}$ es un acto complejo en el que intervienen varias autoridades y se utilizan diversos referentes normativos. Adicional, reitera el alto tribunal, están excluidos del control los vicios materiales, en tanto toda reforma es, per se, contraria al texto anterior y admitir la posibilidad de un control material equivale a la petrificación de la Constitución y a la anulación de la posibilidad de reforma (SC-551, 2003). La cuestión, a partir de tales premisas, se centra en el alcance del control de las reformas a la Constitución, en los términos del art. 379 que lo restringe a los requisitos establecidos en el título XIII y en el significado del concepto "vicios de procedimiento» del art. 241. Los principales elementos dogmáticos expuestos por la Corte Constitucional en la SC-551 (2003) fueron los siguientes: (i) naturaleza de los vicios competenciales, (ii) ausencia de cláusulas pétreas en la $\mathrm{CN}$, existencia de límites materiales al poder de reforma y posibilidades de control y (iii) ámbito del control al poder de reforma.

Sobre el primero de los elementos, recordó la distinción entre vicios de forma, fondo y competenciales. Tal distinción, parte de la base que en todo acto del poder público se distingue la competencia de quien lo dicta, el procedimiento que sigue y el contenido material del acto (Zagrebelsky, 1977: 124). Teniendo en cuenta que en oportunidades anteriores la Corte había considerado que ciertos vicios de competencia eran violaciones materiales de la Carta y no vicios de forma, se anticipa a la posible objeción que se haría al control de esta clase de vicios en el caso de las modificaciones a la $\mathrm{CN}$ argumentando que, en ningún momento, se afirmó que tales vicios fueran exclusivamente vicios de fondo, por cuanto los defectos en la competencia se proyectan sobre el procedimiento y sobre el contenido material. Así, el vicio de competencia tendría una naturaleza binaria, es decir, haría parte de un significado lato sensu tanto del vicio de procedimiento como del vicio de fondo.

No a otra conclusión se llega cuando manifiesta la Corte que, para que un acto jurídico sea válido, es necesario que su contenido se ajuste a la norma superior pero, también, que la autoridad que lo expide adelante el trámite establecido y tenga competencia para ello. Así las cosas, si la competencia es presupuesto del procedimiento no existe obstáculo para que se controlen eventuales vicios de competencia, siempre que estos se proyecten en el procedimiento del acto de reforma. 
Con esto, pasa la Corte a reconocer que no existen dentro de la $\mathrm{CN}$ de 1991 las denominadas cláusulas pétreas ${ }^{10}$ que impidan la plena reforma constitucional. Las cláusulas pétreas son aquellas que prohíben o restringen «la reforma o la modificación de la misma Constitución en que se encuentran insertas» (Ramírez, 2005: 343) ${ }^{11}$. Ahora bien, si no existen cláusulas pétreas, ¿cuál es el fundamento del control de los actos reformatorios de la CN por vicios competenciales? La respuesta es presentada por la Corte en los siguientes términos:

a) en primer lugar, el art. 379 superior no limita el escrutinio de las reformas a la $\mathrm{CN}$ a los vicios de forma, sino que lo extiende a verificar el cumplimiento de los requisitos establecidos en el título XIII;

b) en segundo lugar, la competencia es un presupuesto lógico del procedimiento, careciendo de sentido que se pueda revisar el procedimiento pero se excluya la posibilidad de revisar que el órgano que adelantó la reforma tuviera competencia para hacerla;

c) en tercer lugar, establece que la revisión de los vicios de competencia que se proyectan en el procedimiento se funda en el enunciado del art. 379 superior que ordena verificar el cumplimiento de los «requisitos establecidos en este título», y en la aplicación del principio de legalidad de la función pública previsto en los arts. 6 y 122 .

De esto último se deriva que un poder constituido no puede asumir el rol del poder constituyente por cuanto «estaría minando las bases de su propia competencia».

Finalmente, concluye la Corte que, si bien la $\mathrm{CN}$ no consagra cláusulas pétreas, sí prevé límites a la competencia del poder de reforma. En este sentido sostiene que, en el caso colombiano, existen «límites materiales al poder de reforma del constituyente derivado» que, de ser excedidos, serían el presupuesto lógico del control. Estos límites materiales son conocidos también como límites implícitos, inmanentes o inherentes. Según expresa Loewenstein, «se producen por la inmunidad de que gozan ciertos valores ideológicos funda-

10 En Colombia, la CN de 1821 fue una de las que consagró cláusulas pétreas. Decía el art. 190 lo siguiente: «En cualquier tiempo en que las dos terceras partes de cada una de las Cámaras, juzguen conveniente la reforma de algunos art.s de esta Constitución podrá el Congreso proponerla para que de nuevo se tome en consideración [...]; pero nunca podrán alterarse las bases contenidas en la sección $1^{\text {a }}$ del título I, en la $2^{\text {a }}$ del título II». Cf. Restrepo Piedrahita (2004: 116-117).

11 En el mismo sentido De Vega García (1985: 240). 
mentales» (1970: 192), en un resurgir del iusnaturalismo contra el positivismo jurídico de la Escuela de Viena, se presentan a través de la forma de principios que son, en palabras de García de Enterría, «jerárquicamente superiores» (1984: 99) en la interpretación del texto y, de allí, que supongan un límite a la revisión constitucional, siendo predicables de toda constitución, rígida o flexible y pudiendo ser oponibles, incluso, al propio soberano (Sánchez, 1993: 272-273).

El tercer elemento expuesto por la Corte está referido al ámbito del control al poder de reforma. En este punto, señala el alto tribunal varios aspectos. El primero de ellos es la distinción entre reforma y sustitución. Para la Corte, cuando el art. 374 señala que «La Constitución Política podrá ser reformada [...]», está diciendo, contrario sensu, que no puede ser sustituida.

Lo segundo que señala la Corte son sus propios límites para controlar las reformas a la $\mathrm{CN}$. Expresa que, «el poder constituyente está radicado en el pueblo» y que él mismo, por su carácter originario «no está entonces sujeto a límites jurídicos», lo que trae como consecuencia que, «los actos del poder constituyente originario son fundacionales [...] y por ello dichos actos escapan al control jurisdiccional ${ }^{12}$.

El tercer elemento señalado, es la afirmación según la cual, en el constitucionalismo colombiano, no existe ningún canal de expresión del constituyente originario por cuanto, incluso el referendo, es manifestación de un procedimiento organizado en el que intervienen diversos órganos constituidos.

El cuarto elemento, es que su tesis, en ese momento, se restringe al análisis del referendo como procedimiento de reforma de la $\mathrm{CN}$, no anticipando el tema de los límites materiales cuando se trate de actos legislativos o de Asamblea Nacional Constituyente.

Finalmente, señala la Corte que, entre los límites materiales, están los principios del Estado social de derecho, el carácter republicano del Estado y el sistema presidencial de gobierno.

Luego vendría la SC-1200, $2003^{13}$, en la que reitera la doctrina de la SC-551, 2003 y agrega los siguientes elementos: (i) una distinción más nítida entre la intangibilidad y la insustituibilidad, (ii) las consecuencias del reconocimiento del carácter insustituible de la $\mathrm{CN}$ y (iii) las características del control por vicios de competencia.

Respecto del primer elemento, señala la Corte que, mientras el establecimiento de la Constitución es un acto fundacional, expresión de la soberanía popular, la reforma es un acto de revisión en ejercicio de una competencia atri-

12 En igual sentido Corte Constitucional. SC-544, 1992.

13 Demanda contra los art.s 4 transitorio y 5 (parcial) del Acto Legislativo 3 de 2002. 
buida. En este sentido, está, per se, limitada por el propio acto constituyente. De ello, se desprende que «el poder de revisión no comprende la competencia de derogar o abolir la Constitución».

Así, y para distinguir la intangibilidad de la insustituibilidad, manifiesta la Corte que, mientras el primero impide afectar el núcleo de un principio fundamental, sin importar sus consecuencias dentro de la arquitectura constitucional, la insustituibilidad se traduce en la imposibilidad de alterar el núcleo de un principio que, por sí mismo, transforma la $\mathrm{CN}$ en otra diferente, es decir, la diferencia está signada por las consecuencias sobre la esencia de la Constitución, es decir, que la diferencia es de naturaleza y no de grado.

Sin embargo, como la $\mathrm{CN}$ no tiene cláusulas pétreas, se aborda la pregunta de qué modificaciones se pueden hacer a los principios definitorios. A esto se responde que no son sustituciones parciales las (i) reformulaciones positivas, (ii) reconceptualizaciones, (iii) excepciones específicas y (iv) limitaciones o restricciones.

En segundo lugar, establece la Corte las consecuencias respecto del reconocimiento del carácter insustituible de la $\mathrm{CN}$, tanto frente al cargo presentado por el actor cuando acuse una reforma como inconstitucional por vicios de competencia como respecto de los alcances de la Corte a la hora de controlar la reforma.

Respecto de lo primero, afirma la Corte que el ciudadano tiene la carga de demostrar que hubo una sustitución de la $\mathrm{CN}$, es decir, no basta con (i) argumentar contradicción con cláusula constitucional anterior (antinomia), (ii) ni mostrar que se creó una excepción a otra cláusula constitucional, (iii) ni que «estableció una limitación o restricción frente al orden constitucional anterior». En cualquiera de estos casos, dice la Corte, se trata de un "control material ordinario de la reforma como si ésta fuera inferior a la Constitución».

Frente a lo segundo, se anticipa la Corte a decir que el control de las reformas a la $\mathrm{CN}$ por vicios de competencia genera dos grandes peligros: la petrificación constitucional y el subjetivismo judicial. Por ello, dice la Corte, el método de control de las leyes no puede ser el mismo que el de las reformas a la $\mathrm{CN}$. Mientras que en el primero se confronta el contenido material del acto enjuiciado con los enunciados constitucionales, en el segundo está excluido el control material, debiéndose limitar el juez a evaluar si hubo una sustitución, no en términos formales sino materiales, es decir, si se cambió una Constitución por otra, siendo irrelevante si se viola «un principio preexistente» o si la reforma «es contraria a una regla constitucional».

El tercer aporte dogmático de la SC-1200, 2003, está relacionado con las características del control por vicios de competencia. Lo primero que reconoce la Corte es la necesidad de «delinear un método para determinar cuándo se presenta una sustitución de la Constitución» que evite incurrir en control 
material de la reforma. Reconoce la Corte que dicho método está por ser elaborado y elude su formulación al decir que: «No le corresponde a la Corte delinear dicho método en esta sentencia».

En segundo lugar, sienta la Corte los límites que dicho método de control habrá de respetar so pena de que se desfigure. Así, dice que no se puede (i) mirar la reforma como acto infraconstitucional incapaz de alterar la $\mathrm{CN}$, (ii) transformar cláusulas constitucionales en cláusulas pétreas, (iii) reconocer la supuesta existencia de contenidos normativos supraconstitucionales intocables, (iv) comparar el contenido de la reforma con el contenido de la $\mathrm{CN}$ antes de ésta como si no fuera posible la existencia de antinomias constitucionales y (v) limitarse a señalar la inclusión de excepciones o restricciones sin evaluar si dichas modificaciones trascienden en el ámbito de la reforma y se traducen una sustitución parcial o total.

En tercer lugar, expresa la Corte que, entre los métodos de interpretación que utilizar, el histórico (antecedentes de la reforma) y el sistemático (incluyendo el bloque de constitucionalidad en sentido estricto ${ }^{14}$ ) pueden aportar valiosos elementos de juicio.

\section{TERCERA ETAPA: DE MADURACIÓN, 2004 A 2010}

Con estos antecedentes, empieza la Corte Constitucional a fortalecer su tesis de los límites competenciales, reconociendo, incluso hoy, que se trata de una teoría inacabada (SC-1040, 2005).

En el año 2004, reitera su posición según la cual, quien pretenda acusar una reforma constitucional por vicios de competencia, tiene la carga de demostrar que hubo una sustitución de la Carta (SC-1200, 2003) y en un primer fallo se declara inhibida al considerar que no estaba cumplido dicho requisito $(\text { SC-572, 2004) })^{15}$. Luego $(S C-816,2004)^{16}$, aunque reitera in extenso la SC551,2003 , declara la inconstitucionalidad por vicios de forma sin adentrarse en la posible existencia de un vicio competencial, argumentando que, si bien, «la competencia es un presupuesto del procedimiento», la función del juez constitucional «no consiste en discutir problemas teóricos sino en resolver los casos que les son planteados, dentro de los plazos y condiciones que establece el ordenamiento». En tal sentido, si se vislumbra, a priori, la posible existencia

14 Según la jurisprudencia colombiana, este estaría conformado por «aquellas reglas y principios que, sin figurar expresamente en la Carta, tienen rango constitucional». Cf. Corte Constitucional. SC-228, 2009.

15 Demanda contra el Acto Legislativo 1 de 2003

16 Demanda contra el Acto Legislativo 2 de 2003. 
de un vicio de forma que haga inconstitucional la reforma, puede la Corte, en virtud del principio de «eficiencia procesal», obviar el control de la competencia y proferir decisión de fondo sobre los otros cuestionamientos.

Ese mismo año, en la SC-888 $(2004)^{17}$, reitera que la mayor carga argumentativa frente a las acusaciones por vicios competenciales, está en consonancia «con la exigencia de un mínimo de rigor en la acusación que el ciudadano dirige contra una norma jurídica de rango constitucional ${ }^{18}$. Agrega que, si el actor no presenta razones suficientes pero sí lo hacen los intervinientes, las mismas no se pueden tener en cuenta a la hora de proferir decisión de fondo, por cuanto estas no se pueden considerar "como la demanda que corresponde [...] estudiar» ${ }^{19}$ correspondiendo a la Corte inhibirse por insuficiencia en las razones.

En la SC-970 $(2004)^{20}$, la Corte hace una primera precisión del control de los vicios competenciales y señala lo siguiente: (i) la existencia de límites competenciales se deriva de un análisis sistemático de los distintos procedimientos de reforma, partiendo del art. 374; (ii) el art. 376 señala tres límites expresos en el caso de la Asamblea, a saber: la ley que convoca debe prever la competencia, el periodo y la condición de quedar en suspenso la facultad constituyente del Congreso, y (iii) la distinción entre poder constituyente primario del pueblo y poder constituido y la «ausencia de una habilitación para la sustitución de la Constitución». Con estos elementos, dice la Corte, se sustenta el control constitucional de las reformas a la $\mathrm{CN}$ por vicios de competencia.

Continúa la Corte reiterando que la sustitución es el cambio de una constitución por otra, pero agrega que, en este caso, para que sea válida se debe tratar de un "cambio de su fuente de legitimidad" y tal circunstancia se da cuando «la nueva Constitución no pueda tenerse como la continuación de la anterior», sino que es producto de un nuevo acto constituyente.

Lo más relevante en este pronunciamiento, es la formulación del primer silogismo jurídico, el que, si bien sería complementado tiempo más tarde, sirvió de base para la construcción doctrinaria del actual juicio de sustitución ${ }^{21}$.

17 Demanda contra el numeral 3 (parcial) del art. 3 del Acto Legislativo 3 de 2002.

18 En igual sentido Corte Constitucional. SC-1124, 2004.

19 La Corte Constitucional, en la SC-757, 2008, justificaría esta posición en el carácter rogado de la $\mathrm{AI}$ y en el principio de autocontención judicial.

20 Demanda contra el inciso segundo del art. 4 transitorio del Acto Legislativo 3 de 2002.

21 Como diría tiempo después, las etapas de este juicio «lo distinguen del juicio de intangibilidad y del juicio de violación de un contenido material de la Constitución». Corte Constitucional. SC-1040, 2005. 
Los elementos metodológicos estarían dados por el elemento definitorio (premisa mayor), el elemento normativo enjuiciado (premisa menor) y el resultado de la comparación (comparación) ${ }^{22}$.

Un año más tarde, mediante SC-1040, 2005²3, se delinearían los pasos para diferenciar la reforma de la sustitución. Por un lado, tendríamos el método y, por otro, el resultado. Frente al método, es necesario determinar que se está frente a un principio definitorio, es decir, la construcción de la premisa mayor en el juicio de sustitución.

Con esta delimitación del principio definitorio o premisa mayor, se procede a compararlo con la reforma para determinar si «dicho elemento esencial definitorio ha sido (vi) reemplazado por otro — no simplemente modificado, afectado, vulnerado o contrariado- y (vii) si el nuevo elemento esencial definitorio es opuesto o integralmente diferente, al punto que resulte incompatible con los elementos definitorios de la identidad de la Constitución anterior».

Frente al resultado, manifestó la Corte que, el mismo «debe poder llevar a la conclusión de que, como el elemento esencial definitorio ha sido remplazado por otro opuesto o integralmente diferente, no es posible armonizar la reforma constitucional con el resto de normas constitucionales que no fueron modificadas por ella y que reflejan aspectos claves de lo insustituible». A esto se agrega que en la evaluación no solo se toman en cuenta las normas contenidas en el texto de la Constitución, sino también todas aquellas que se le integran a través de la figura del bloque de constitucionalidad ${ }^{24}$.

Finalmente, señala la Corte un nuevo concepto de sustitución, al decir que «el concepto de sustitución refiere a una transformación de tal magnitud y trascendencia, que la Constitución anterior a la reforma aparece opuesta o integralmente diferente a la que resultó después de la reforma, al punto que ambas resultan incompatibles».

En el año 2006, se consolidan los anteriores elementos jurisprudenciales. En la SC-181, $(2006)^{25}$, manifestó que en el juicio de sustitución no se controla el caos, el desorden, la inconveniencia que puede provocar la aplicación de un AL, ni las posibles antinomias constitucionales. En la SC-472 (2006) ${ }^{26}$, se inhibe por ausencia de razones suficientes y admite, sin eufemismo alguno, que el juicio por vicios de competencia involucra «la revisión material de un

22 Criterios reiterado por la Corte Constitucional en las SC-971, 2004 y SC-1124, 2004.

23 Demanda contra el Acto Legislativo 2 de 2004.

24 En el mismo sentido, Corte Constitucional SC-1040, 2005.

25 Demanda contra del contra el Acto legislativo 1 de 2005.

26 Demanda contra el art. 1 (parcial) del Acto Legislativo 1 de 2005. 
Acto Legislativo cuando dicho acto comprenda una sustitución de la Constitución $»^{27}$. En la SC-740 (2006) ${ }^{28}$ agrega que no es posible, en un juicio de sustitución, hacer uso del principio de proporcionalidad ni comparar el contenido de la reforma con el contenido de los tratados de los cuales Colombia es parte, así estos integren el bloque de constitucionalidad, por cuanto «ello implicaría un juicio material». Finalmente, en la SC-986 (2006) ${ }^{29}$ se inhibe por insuficiencia en las razones y se limita a reiterar la doctrina anterior.

El año 2007 es también un año de reiteración, salvo la SC-153 (2007) ${ }^{30}$, en la que dice que el cargo de sustitución debe estar lo suficientemente estructurado para evitar un subjetivismo judicial que impida injustificadamente la evolución constitucional y determine la existencia de una presunta sustitución y que, en virtud de la distinción entre poder constituyente primario y poder constituido, la sustitución solamente se puede adelantar, en el caso colombiano, por el constituyente originario o por una Asamblea Nacional Constituyente elegida para ello. Es esta la primera vez que la Corte acepta que el procedimiento de la Asamblea es una excepción al art. 374 y que constituye un mecanismo apropiado para la sustitución constitucional. Finalmente, en las SC-178 (2007) ${ }^{31}$ y SC-293 (2007) $)^{32}$, no existe aporte alguno.

En el año 2008, mediante la SC-757 (2008) $)^{33}$, se reiteran los lineamientos anteriores sobre el juicio de sustitución, en especial los sentados en la SC1040 (2005) y la SC-888 (2004).

Los años 2009 y 2010 terminarían por consolidar la tesis de los límites competenciales al poder de reforma. Sin desconocer que la sustitución es un concepto no acabado, estos dos últimos años servirían para introducir los elementos finales que hoy sustentan en buena medida la aplicación de dicha

27 En igual sentido, Corte Constitucional SC-720, 2006. En la misma línea argumental, había dicho la doctrina que: «La tesis de los vicios competenciales implica, en la práctica, un control material de la reforma, del tipo implícito textual, en la medida en que es la Corte quien determina los sentidos de la Constitución que no permiten ser reemplazados por la reforma del Congreso. Cabe anotar, que pese a ello, la Corte no acepta que esté realizando un control material». Cf. Cajas Sarria ( ):30.

Demanda contra el Acto Legislativo 1 de 2005.

29 Demanda de inconstitucionalidad contra el art. 1 (parcial) del Acto Legislativo 1 de 2005.

30 Demanda de inconstitucionalidad contra el art. 1 (parcial), el parágrafo 2 del art. 1 y los parágrafos transitorios 2 y 3 del art. 1 del Acto Legislativo 1 de 2005 .

31 Demanda contra los arts. 1 y 2 del Acto Legislativo 1 de 2005.

32 Demanda contra del Acto Legislativo 1 de 2005.

33 Demanda contra el Acto Legislativo 1 de 2007. 
tesis. Así, mediante la SC-588 (2009) $)^{34}$, la Corte expresa que la reforma, no solo puede girar sobre una adición al contenido anterior sino que, incluso, puede llevar a la adopción de "tesis enfrentadas», sin que sea posible la "toma de partido por alguna de las opciones en pugna [...], como si fuera imposible cualquier contacto o relación entre ellas" por cuanto ella se ve "precisada a compararlos», pues no de otra manera es posible determinar si hubo una reforma o una sustitución, aclarando que la comparación no es entre el texto anterior y el nuevo, sino entre la Constitución anterior y la que surge de la reforma.

Uno de los elementos más importantes introducidos en esta sentencia es la diferencia entre reforma, destrucción, supresión, quebrantamiento y suspensión de la Constitución. Para la Corte, «la destrucción de la Constitución se produce cuando se suprime la Constitución existente y esa supresión está acompaña de la del poder constituyente en que la Carta se basaba». Dicha situación se puede presentar cuando se subvierte el orden constitucional mediante la sustitución del titular del poder constituyente, como lo es cuando se pasa de una monarquía absoluta a una democracia, o en el caso del golpe de Estado donde «la ruptura del orden es absoluta y de su obra no cabe resquicio de continuidad jurídica» ${ }^{35}$.

La supresión, por su parte, "conserva el poder constituyente en que ésta se basaba, aunque también se produce resquebrajamiento de la continuidad jurídica, porque se suprime la Constitución, como acaece siempre que, en ejercicio del poder de reforma, se dé lugar a un cambio total de la Carta». Este cambio, sin embargo, se da en términos materiales. De este modo, aunque el poder constituyente es el mismo, actúa como si fuera uno distinto por fuera de los límites existentes en el anterior sistema constitucional ${ }^{36}$.

En el caso del quebrantamiento, estamos ante «la violación de prescripciones constitucionales», a título excepcional y para unas situaciones específicas, adelantado bien mediante procedimientos autorizados por la $\mathrm{CN}$ o mediante el uso de figuras extraordinarias no previstas constitucionalmente ${ }^{37}$, esto es, parafraseando a Schmitt, un quebramiento constitucional de la Cons-

34 Demanda contra el art. 1 del Acto Legislativo 1 de 2008. Mediante Auto 281, 2010, la Corte Constitucional resolvió negativamente la solicitud de nulidad contra la SC588, 2009.

35 Cfr. García-Atance (2002: 78 y 79).

36 Para la Corte esto constituye una sustitución total.

37 A juicio de la Corte Constitucional, las excepciones específicas no constituyen sustitución parcial de la Carta. La diferencia entre dicha excepción y el quebrantamiento de la CN estaría dado por la magnitud de la excepción. Así, cuando (i) la excepción se aplica a todo el supuesto protegido en la norma, (ii) no se siguen los trámites previstos 
titución y un quebrantamiento inconstitucional de la misma (1982: 116). Así, según señala la doctrina, "puede preverse en la Constitución, en modo detallado o con normas de reenvío a especificaciones sucesivas, pero puede darse también una omisión de consideración o incluso una prohibición textual» (De Vergottini, 2004: 178). Por ejemplo, cuando se toman medidas de facto o dictatoriales como el cierre de un ente público basado en normas abiertas de la $\mathrm{CN}^{38}$. Esa supresión, expresa la doctrina, es válida cuando es prevista $a b$ initio por la $\mathrm{CN}$ o cuando posteriormente es necesaria, siempre que la necesidad se señale expresamente en el texto constitucional (Sánchez, 1993: 269). También es necesaria cuando se presentan situaciones de facto, no previstas ni reguladas en la $\mathrm{CN}$, es decir, la suspensión no encuentra fundamento «en normas formales de la Constitución, si no en el principio de necesidad y, por ende, en una fuente-hecho» (De Vergottini, 2004: 178).

Finalmente, la suspensión ocurre cuando una o varias disposiciones constitucionales quedan sin vigencia de manera temporal, bien porque mediante una norma especial se suspenden los efectos de la disposición constitucional ora porque se prevé una excepción temporal a la aplicación del precepto. Esa suspensión se puede presentar en observancia a los procedimientos constitucionales o en violación de ellos ${ }^{39}$.

A juicio de la doctrina (Sánchez, 1993: 269), la suspensión de las disposiciones constitucionales puede ser, igualmente, directa o indirecta, según que se aluda o no a ellas en el contenido de la norma que prevé la suspensión ${ }^{40}$. Este último supuesto, concluye la Corte, se traduce en una sustitución tácita que, en tal sentido, debe ser objeto de control además de contrariar, sin lugar a dudas, la naturaleza misma de las normas transitorias, cuyo objeto es «facilitar la aplicación definitiva de una regulación nueva ${ }^{41}$ y no entrar a suspender otras ya existentes y así, aunque se afirme que la reforma es transitoria,

para la reforma, (iii) carece de justificación o (iv) se afectan varios principios de los cuales ni siquiera se hace alusión, estamos ante un quebrantamiento.

38 Otros serían, la prolongación, mediante reforma de la $\mathrm{CN}$, del periodo del Parlamento mediante ley ordinaria, cuando la duración del mandato está prevista en la CN. Cf. Sánchez Ferriz (op. cit.: 270).

39 El profesor Sagües explica que, la suspensión puede ser total y parcial y que, como nota característica, las disposiciones constitucionales permanecen válidas mientras rige el «ordenamiento de excepción». Sagües (2001: 351-352).

40 Por ejemplo, la Corte Constitucional en la SC-588, 2009, concluyó que la suspensión del art. 125 sobre carrera administrativa, suspendía el derecho de igualdad (art. 13), el derecho político de acceso a la función pública a través del mérito (art. 40-7) y la separación de poderes (art. 113).

41 Cf. Gretel (1986: 155). Citados por Corte Constitucional. SC-901, 2008. 
«sus normas generarían situaciones jurídicas definitivas para sus destinatarios» (SC-901, 2008)

El año 2010, con el cual se cierra esta tercera etapa, culminaría con las SC-303 y SC-397. En la SC-303, 2010 ${ }^{42}$, se discute la posibilidad de conocer de demandas contra reformas a la Constitución de carácter transitorias que perdieron vigencia antes o después de la puesta en marcha del control constitucional. Si bien es cierto, en la SC-588, 2009, ya se había dicho que, si esas normas transitorias generaban una suspensión constitucional, era procedente el control por vicios de competencia, en esta oportunidad se aclara que si las normas desparecieron por haber agotado sus efectos se presenta el fenómeno de la sustracción de materia por carencia actual de objeto ${ }^{43}$. Al contrario, si la demanda se presenta durante el tiempo de vigencia de la norma, en aplicación al principio de perpetuatio jurisdictionis, es posible continuar con el análisis de la demanda. Son dos las razones de esta situación: por un lado, el contenido del derecho de acceso a la administración de justicia, el cual se vería lesionado si al actor, se le obligara a «asumir las consecuencias del paso del tiempo durante el trámite ante la Corte», pese a la oportunidad de su intervención. Por otro lado, el carácter jurisdiccional de la función desarrollada por la Corte, la cual se vería alterada con la posibilidad de que existieren normas excluidas del control constitucional. Luego de un recuento de algunos de los pronunciamientos de la Corte en esta materia, resume el alto tribunal los mecanismos de restricción del análisis por vicios de competencia, con lo cual se busca evitar un «desbordamiento de las competencias de esta Tribunal». Esos elementos son: «[...] la cualificación de la acción pública de inconstitucionalidad, la necesidad de conservar la precisión conceptual sobre la materia y la sujeción a una metodología particular para adelantar el juicio de sustitución». Frente a la cualificación, está la exigencia al ciudadano actor de una mayor carga argumentativa en la que se demuestre que se sustituyó la $\mathrm{CN}$; respecto de lo segundo, reitera la exigencia al tribunal de no confundir el control por vicios de competencia con control material de las reformas a la $\mathrm{CN}$, en los términos previstos desde la SC, 1200, 2003; finalmente, la necesidad de aplicar un silogismo jurídico en el control de las reformas a la $\mathrm{CN}$ por vicios de competencia, según se expuso en las SC-970, 2004 y SC-1040, 2005.

42 Demanda contra el parágrafo transitorio 1 del art. 1 del Acto Legislativo 1 de 2009.

43 En igual sentido, Corte Constitucional SC-757, 2004; SC-992, 2001; SC-1373, 2000; SC-685, 1996; SC-350, 1994. 
En la SC-397, 2010 ${ }^{44}$, hay tres elementos relevantes: (i) reitera, de conformidad con las SC-551, 2003 y SC-141, 2010, que el referendo, inclusive de origen ciudadano, no es manifestación del constituyente primario, en tanto se trata de un trámite complejo adelantado según un procedimiento determinado; (ii) que el Congreso de la República está autorizado para introducir modificaciones al proyecto de ley de origen ciudadano, siempre que no sean modificaciones sustanciales, lo que reforzaría, aunque así expresamente no lo diga la Corte, la tesis de que se trata de la expresión de un poder constituido, por lo menos, en la convocatoria del referendo, y (iii) señala las hipótesis que constituyen modificación sustancial. Con esto se cierra la tercera etapa, la de maduración, y se pasa a la cuarta y actual etapa de reiteración o reafirmación de las tesis.

\section{LA CUARTA Y ACTUAL ETAPA ES DE APLICACIÓN DE LA TESIS: REAFIRMACIÓN}

En el año 2011, la SC-574, 2011, reitera que la competencia es un presupuesto lógico del procedimiento (SC-551, 2003) y sistematiza, una a una, las sentencias de la Corte sobre esta temática. Es, en palabras de la doctrina, una sentencia consolidadora de línea ${ }^{45}$. Al final, sintetiza en ocho puntos los lineamientos del control constitucional de las reformas a la $\mathrm{CN}$ por vicios de competencia ${ }^{46}$.

44 Revisión de constitucionalidad de la Ley 1327 de 2009, «por la cual se convoca a un referendo constitucional y se somete a consideración del pueblo un proyecto de Reforma Constitucional».

45 Un explicación de este concepto en López Medina (2009: 33).

46 Señala la Corte lo siguiente: «1. Que por tratarse de una demanda por un vicio formal relacionada con la competencia, la Corte tiene que verificar que no se haya sobrepasado el término de caducidad de un año [...]. 2. Que el juicio de sustitución no es un juicio de intangibilidad ni tampoco un juicio de un contenido material de la Constitución [...]. 3. Que el concepto de sustitución no es un concepto completo, acabado o definitivamente agotado que permita identificar el conjunto total de hipótesis que lo caracterizan (...). 4. Que la sustitución de la Constitución puede ser total, parcial, temporal o definitiva [...]. 5. Que para determinar si la Constitución fue sustituida por otra - parcial, total, transitoria o permanentemente - se debe realizar el llamado ‘juicio o metodología de la sustitución’ que está compuesto de tres premisas [...]. 6. Que para la verificación del cumplimiento de la Premisa mayor, la Corte debe comprobar a través de una lectura transversal e integral de la Constitución de 1991 [...]. 7. Que mediante el llamado test de la eficacia el juez constitucional puede comprobar: (i) si las normas constitucionales a reformar siguen siendo las mismas antes y después 
En el año 2012, en las SC-170 ${ }^{47}$, SC-574, 2011, SC-249, 2012 ${ }^{48}$, SC$317,2012^{49}$ y SC-846, 2012 $2^{50}$, se limita a reiterar la doctrina anterior, el carácter definitorio de algunos principios y sistematizar algunos actos que no se tienen como vicios de competencia.

Por último, en la SC-433, 2013 ${ }^{51}$, la Corte se inhibe de conocer de la demanda por ineptitud sustantiva de la demanda. Sin embargo, los salvamentos de votos dejan en evidencia las debilidades dogmáticas de aplicación de los mismos lineamientos de la jurisprudencia sobre la procedencia de las demandas contra actos reformatorios de la $\mathrm{CN}$, lo cual lleva a presumir que esta cuarta etapa será de reafirmación y refinamiento de las tesis expuestas ${ }^{52}$.

\section{UNA MIRADA CRÍTICA DESDE LA DISCRECIONALIDAD JUDICIAL}

\section{LA DISCRECIONALIDAD JUDICIAL}

En el lenguaje común, el término «discreción» es un concepto polisémico que, aunque conduce hacia un mismo sentido, puede presentar diferentes alcances según sea lo que se está discutiendo. Desde un punto de vista gramatical, bien puede expresar «sensatez» o «prudencia» como «arbitrio» y «voluntad $»^{53}$. En el lenguaje jurídico, igualmente se generan enormes dificul-

de la reforma [...], (ii) que el cambio no dio lugar a que se establecieran normas ad hoc o particulares, y (iii) que no se hayan sustituido tácitamente a través de la reforma otros principios estructurales de la Constitución, dando lugar al fraude de la Constitución. 8. Teniendo en cuenta lo anterior se puede afirmar que dentro de la clasificación de los límites al poder de reforma constitucional, la Corte Constitucional a través de la teoría de la inconstitucionalidad por sustitución ha reconocido que existen unos límites intrínsecos al poder de reforma, ya que estos se encuentran reflejados en la Constitución misma o en los elementos del bloque de constitucionalidad» (negrita en el texto).

47 Demanda contra el Acto Legislativo 2 de 2011.

48 Demanda contra el Acto Legislativo 4 de 2011.

49 Demanda contra el Acto Legislativo 5 de 2011.

50 Demanda contra el Acto Legislativo 1 de 2011.

51 Demanda contra el Acto Legislativo 6 de 2011.

52 Como lo expresa la doctrina, "los test de razonabilidad y la metodología que se utiliza para resolver las impugnaciones de la demanda son confusas y ambiguas, ya que parte de una axiología analítica cuestionable, que facilita el 'subjetivismo judicial' en la decisión». Cf. Ramírez Cleves (2006: 24).

53 Tomado de www.rae.es, consultado el 31/08/2013. 
tades conceptuales. Por ello, y para los fines del presente ensayo, se limitará la utilización del concepto a la función jurisdiccional por parte de los tribunales y, claro, enfocada en la aplicación de la teoría de los límites competenciales al poder de reforma de la $\mathrm{CN}$ de 1991 por parte de la Corte Constitucional.

Podría decirse que, según una concepción generalizada, existen cuatro sentidos del vocablo "discrecionalidad judicial», del cual ahora nos interesan tan solamente dos: la discreción débil y la discreción fuerte ${ }^{54}$. Primero, frente a la discreción débil, vista como la «sensatez y juicio para adoptar el curso de acción jurídicamente previsto» (Iglesias Vila, 1999: 25), tenemos que ella alude al hecho de que la solución a ciertos problemas, aun cuando prevista en el ordenamiento jurídico, no resulta obvia. A este respecto, Dworkin (1984: 84) manifiesta que la discreción débil opera en aquellas hipótesis en las que las normas no se pueden aplicar mecánicamente, exigiendo algún grado de discernimiento. En este sentido, la discreción débil se distingue por estar en "presencia de una solución correcta cuya identificación requiere una dinámica interpretativa donde se ponen a prueba las capacidades intelectivas del intérprete» (Iglesias Vila, 1999: 64).

Segundo, frente a la discreción fuerte, vista como «la posibilidad de elección entre diferentes cursos de acción igualmente válidos o admisibles» (Iglesias Vila, 1999: 28), tenemos que la misma opera en situaciones en las que existe (i) un elemento externo u objetivo, visto como la ausencia de respuesta correcta, lo que obliga a la escogencia de entre distintas alternativas; (ii) un elemento subjetivo, entendida como la creencia interna del agente de que no existe una única respuesta, y (iii) un elemento procedimental, evidenciado en la posibilidad y efectiva escogencia de una de dichas alternativas (Iglesias Vila, 1999: 60).

Lo anterior nos lleva a concluir anticipadamente que la discreción judicial fuerte es, per se, un concepto limitado. En este sentido, desde una concepción positivista del derecho, esta figura no comprende ni las decisiones arbitrarias — entendiendo por tal aquellas que van contra la evidencia, i.e., afirman la existencia de distintas opciones cuando existe solo una- ni aquellas que partan de creencias erróneas.

Las anteriores consideraciones llevan a relacionar, desde el positivismo jurídico, el concepto de discreción fuerte con el de caso difícil. Desde esta perspectiva, la discreción fuerte se da un contexto de indeterminación parcial

54 Los dos sentidos que se dejan por fuera son los siguientes: por un lado, discreción fáctica, esto es, frente a la fijación de los hechos relevantes en un proceso y, por otro, discreción funcional, en tanto la decisión judicial no está sujeta a revisión. Cf. Iglesias Vila (1999: 27 y 28). 
del derecho, la cual conduce a un margen más o menos amplio de libertad para la toma de la decisión. Podríamos decir, siguiendo a Hart (2004: 157158), que la ausencia de una respuesta correcta — en el sentido de que no hay más respuestas correctas-, es un rasgo característico de los casos difíciles, esto es, de hipótesis de discreción fuerte.

Por lo anterior, resulta imperioso delimitar el ámbito de lo que, desde el positivismo, se entiende o se puede entender por caso difícil para, de este modo, señalar el ámbito de la discreción fuerte. Así, existen: (i) casos normativamente difíciles, que son aquellos que carecen de repuesta normativa, es decir, que no tienen una calificación deóntica ${ }^{55}$; (ii) casos epistémicamente difíciles, que son aquellos en los que, pese a la existencia de una respuesta jurídica, se requiere de un esfuerzo intelectual para su identificación y aplicación, i.e., una $\mathrm{CN}$ que si bien garantiza el derecho a la conformación de una familia no precisa si únicamente se pueden conformar a partir de relaciones heterosexuales o si caben igualmente las parejas del mismo $\operatorname{sexo}^{56}$; (iii) casos pragmáticamente difíciles, que se presentan cuando la solución se preña de situaciones externas que trascienden a la decisión, i.e., como cuando la Corte Constitucional colombiana tuvo que determinar si la inviolabilidad de los congresistas cubría la función judicial, en un momento en que no hacerlo habría significado la sanción penal para aproximadamente 110 congresistas $^{57}$; (iv) casos fácticamente difíciles, que surgen cuando existen supuestos tan complejos que no permiten determinar si un hecho reviste de ciertas circunstancias, i.e. lo que sucede con la figura del Estado de cosas inconstitucional ${ }^{58}$. (v) finalmente, casos moralmente difíciles, que se dan cuando la respuesta normativa lleva a casos injustos o de difícil justificación moral ${ }^{59}$.

En contextos como los anteriores, se justifica la existencia de una discrecionalidad fuerte y, sobre estas y otras ideas conexas que se irán presentado, se intentará demostrar que en la construcción dogmática de la tesis de los límites competenciales, se pasó de un ámbito de discreción débil —donde había o

55 Para la Corte Constitucional de Colombia, en la medida en que la función ontológica del juez es «fallar», tiene que hacerlo aun cuando no exista solución desde el derecho normativo para lo cual puede recurrir a las fuentes del derecho. Cf. Corte Constitucional, SC-085, 1995. Esta situación se puede presentar, entre otras razones, por la existencia de lagunas, antinomias o indeterminaciones normativas.

56 Véase Corte Constitucional, SC-577, 2011.

57 Véase Corte Constitucional, SU-047, 1999.

58 Sobre esta figura en Colombia véase Corte Constitucional, ST-025, 2005.

59 Por ejemplo, una decisión de la Corte donde estableció que la existencia de regímenes pensionales especiales contrariaba valores constitucionales. Cf. Corte Constitucional, SC-258, 2013. 
creemos había una respuesta correcta- a una discreción fuerte —en la que se eligió de distintas opciones, planteadas por la Corte, pero sin un sustento en el ordenamiento jurídico-, y de una discreción fuerte — donde efectivamente existían diversas opciones - a una zona de arbitrariedad —en tanto se hizo uso de soluciones, sino no existentes por lo menos no evidentes- para terminar justificando unas decisiones que se podrían explicar más fácilmente desde la ciencia política o la sociología política o jurídica que desde la teoría del Estado o el propio derecho constitucional.

\section{DE LA DISCRECIÓN DÉBIL A DISCRECIÓN FUERTE: CONTROL DE VICIOS DE COMPETENCIA Y LA DISTINCIÓN ENTRE REFORMA Y SUSTITUCIÓN}

De las múltiples críticas que podría suscitar la construcción dogmática de la teoría de los límites competenciales al poder de reforma de la $\mathrm{CN}$ de 1991, hay dos que merecen especial reparo y que se pueden analizar desde el ejercicio de la discreción judicial. El primer caso, se evidencia con el uso que hace del concepto de «vicios de competencia» y, el segundo, con la posibilidad de que la Corte ejerza control sobre dichos vicios.

Frente a la primera crítica, tenemos que la Corte pasó de una hipótesis de discreción débil —en tanto existía una regla que proveía una sola respuestaa una discreción fuerte, donde se ofrecen distintas posibilidades de decisión, todas igualmente válidas. Sin embargo, cuando se abre paso a esta opción, a partir de la configuración en una zona de penumbra que en principio no existía, se fijan como posibles algunas soluciones que, aplicando los métodos de Hart y de Dworkin para la solución de casos difíciles, no se desprendía de los elementos teóricos expuestos por la Corte $^{60}$.

La primera hipótesis de discreción para la Corte — que nosotros consideramos era un ámbito de discreción débil— está relacionada con la competencia para controlar las reformas a la CN. Así lo expresa el art. 241 de la CN: "A la Corte Constitucional se le confía la guarda de la integridad y supremacía de la Constitución, en los estrictos y precisos términos de este artículo [...]».

60 Si bien es cierto que los tribunales constitucionales ostentan una autonomía procesal, también lo es que el ámbito de la misma se restringe a la existencia de tres elementos: (i) la decisión se dé en un caso constitucional concreto, (ii) verse sobre cuestiones puramente procesales y (iii) se trate de asuntos que no puedan resolverse con los medios tradicionales de creación judicial del derecho. Es decir, no se puede alegar esta potestad para sustentar el ejercicio de una discreción fuerte, casi arbitraria, como la que se expresa en la tesis de la Corte. Cf. Moreno Millán (2007). 
De entrada, la $\mathrm{CN}$ está fijando que se trata, como toda función pública, de unas competencias asignadas en forma taxativa, no habiendo lugar a interpretaciones extensivas que permitan el ejercicio de atribuciones no expresamente señaladas o que no correspondan a "presupuesto lógico» de la materia. Seguidamente, cuando fija el ámbito de las competencias del control constitucional frente a las reformas a la $\mathrm{CN}$ por las vías del acto legislativo, del referendo y de la Asamblea Nacional Constituyente expresa, para todos los casos, que el control es «solo por vicios de procedimiento en su formación». El adverbio «solo», se traduce en la exclusión de cualquier otro elemento que no haga parte o esté intrínseco en el concepto de "vicio de procedimiento».

Sin embargo, pese a tan literal restricción, la Corte afirma que el vicio de competencia, que es un "presupuesto lógico del procedimiento", se proyecta no solamente al trámite surtido ante las cámaras y ante los demás entes intervinientes, sino también al contenido mismo de la reforma. La anterior afirmación surge de lo establecido en el art. 374 de la CN, el cual expresa que: «La Constitución Política podrá ser reformada por el Congreso, por una Asamblea Constituyente o por el pueblo mediante referendo». Expresa la Corte que, si el artículo utiliza el verbo «reforma», está limitando la competencia del poder constituyente derivado, el cual no podrá sustituir la Constitución por otra, ni de manera parcial ni de manera total.

Así, para la Corte, la regla — podríamos afirmar que las normas de asignación de competencia, salvo disposición en contrario son reglas- del art. 241 se complementa con las reglas de los artículos 374 y 379. Este último expresa que: "Los Actos Legislativos, la convocatoria a referendo, la consulta popular o el acto de convocación de la Asamblea Constituyente, solo podrán ser declarados inconstitucionales cuando se violen los requisitos establecidos en este título».

Hasta aquí la tesis no tendría mayor objeción de no ser por lo siguiente: las reglas expresan supuestos de todo o nada (Dworkin, 1984: 24). Así, cuando la regla del art. 241 establece que, el control será «Solo por vicios de procedimiento en su formación", está excluyendo cualquier otra consideración al respecto. Se podría contraargumentar, siguiendo a la Corte, que la competencia es un "presupuesto lógico del procedimiento" y que, cuando el art. 379 nos dice que las reformas «solo podrán ser declarados inconstitucionales cuando se violen los requisitos establecidos en este título», y en los términos del art. 374 la CN solo puede ser reformada, excluyendo la hipótesis de la sustitución, se habilita el ejercicio de un control material o de contenido de que permita verificar si la modificación de la $\mathrm{CN}$ conduce a una reforma —en el sentido de que son se alteran sus elementos esenciales - o si se traduce en una sustitución —entendiendo que la CN pasa a ser otra después de la reforma. 
Sin embargo, tal salida encierra el paso de un ámbito de discreción débil, donde existía una respuesta correcta, no evidente, pero obtenible a partir de un ejercicio hermenéutico comprensivo, a una zona de penumbra donde la Corte tiene la posibilidad de escoger entre diversas opciones. Veamos por qué se dio tal paso.

Supongamos que la regla del art. 241, se complementa con las reglas del art. 374 y del art. 379. Según Dworkin (1984: 24-25), cuando una regla interfiere en el ámbito de otra, o la sustituye o genera una excepción específica. Esto significa que una regla no necesariamente excluye a la otra sino que puede haber una simbiosis de aplicación paralela en circunstancias específicas. La pregunta sería: ¿cómo se soluciona un caso de difícil interpretación? Es decir, ‘hacia dónde se debe dirigir la interpretación para determinar si la deroga o si la complementa y, en este caso, cuál es el supuesto de excepción? Al respecto, Hart (2004: 159) manifiesta que la existencia de un caso difícil se puede solucionar a partir de la analogía, esto es, de la verificación de si se trata de una semejanza en aspectos relevantes. La técnica que utilizar entonces, es el acercamiento a conceptos semejantes o "presupuestos lógicos». En un sentido no muy lejano, Dworkin manifestará que la «teoría de la adjudicación» posibilita la obtención de una respuesta correcta, aún en presencia de un caso difícil (hard cases), desde una visión sistemática o global del sistema jurídico y con una «justificación moral» de ese derecho (Vigo, 2006: 49).

Para el caso concreto, no está en discusión la necesidad de evaluar la competencia como presupuesto del procedimiento. Dicho de otro modo, la pregunta acerca de si se podía evaluar la competencia, no estaba en un ámbito de discreción fuerte, porque era un elemento intrínseco al procedimiento. Asimismo, la pregunta de cuál es el ámbito de la competencia que puede ser controlado por la Corte también pasa por una discreción débil, en tanto el concepto de "competencia» está signado por la adscripción formal de una función.

En el caso de las reformas a la $\mathrm{CN}$, la competencia está regulada en diversos artículos que delimitan el ámbito de verificación de la Corte. Así, frente a los actos legislativos, nos dice el art. 375 que: «Podrán presentar proyectos de acto legislativo el Gobierno, diez miembros del Congreso, el veinte por ciento de los concejales o de los diputados y los ciudadanos en un número equivalente al menos, al cinco por ciento del censo electoral vigente».

Respecto del referendo, prevé el art. 378 que: «Por iniciativa del Gobierno o de los ciudadanos en las condiciones del artículo 155». Y sobre el tema de la Asamblea Nacional Constituyente, nos dice el art. 155 que: «Podrán presentar proyectos de ley o de reforma constitucional, un número de ciudadanos igual o superior al cinco por ciento del censo electoral existente en la fecha respectiva o el treinta por ciento de los concejales o diputados del país». 
Es en el ámbito de verificación de que la propuesta haya sido presentada por los autorizados y de que la reforma o la ley que convoca a la misma se haya tramitado por el Congreso donde se realiza el control de competencia. ¿La razón? Porque la analogía que pregona Hart se hace a partir de un acercamiento a los casos fáciles (Rodríguez, 1997: 34), es decir, en el núcleo de la norma que se aplica. Así, está más cerca el concepto de vicio de competencia del tema preciso de a quién se le permite presentar proyecto de reforma y quién la tramita (vicio de procedimiento) que del tema de si la modificación es una reforma o una sustitución, para lo cual, necesariamente, se debe revisar el contenido de la modificación (vicio de fondo).

Esta situación, sin lugar a dudas, lleva a la Corte de una discreción débil — donde la única respuesta correcta se daba en el sentido de que a la Corte le correspondía verificar la competencia para iniciar, adelantar o culminar el procedimiento - hacia una zona de penumbra con discreción fuerte — donde ella evalúa si el contenido de la modificación es reforma o sustitución, es decir, donde escoge de dos opciones posibles-, con el agravante que no solo contraría postulados axiomáticos del derecho constitucional — como aquel según el cual las reformas a la $\mathrm{CN}$ no se pueden controlar por vicios de fondosino que incluso reconoce expresamente que la revisión de vicios de competencia implica un control material de la reforma ${ }^{61}$.

Se podría alegar que, en el caso de los límites implícitos al poder de reforma de la $\mathrm{CN}$, la salida teóricamente posible es la de aceptar que el poder constituido no la puede sustituir por cuanto minaría «las bases de su propia competencia ${ }^{62}$. Sin embargo, ante esta objeción teórica, está la razón normativa del art. 241: solo por vicios de procedimiento en su formación.

Es así como, en el desarrollo de la tesis de los límites competenciales, se pasó de una discreción débil a una fuerte — diciendo que la competencia es presupuesto lógico del procedimiento- y se entró a escoger de entre dos posibles opciones - una según la cual era competencia para iniciar, tramitar o culminar el procedimiento, a otra según la cual la competencia estaba enmarcada en los conceptos «reforma-sustitución»-, con lo que la discreción fuerte pasó a una zona de arbitrariedad —en el entendido que la $\mathrm{CN}$ restringió

61 Así, la Corte Constitucional expresó en la SC-472, 2006, que el control implica, «la revisión material de un Acto Legislativo cuando dicho acto comprenda una sustitución de la Constitución».

62 En este sentido, se ha dicho que: «La facultad de reformar la Constitución contiene, pues, tan solo la facultad de practicar, en las prescripciones legal-constitucionales, reformas, adiciones, refundiciones, supresiones, etc.; pero manteniendo la Constitución; no la facultad de dar una nueva Constitución». Schmitt, (1982: 119). 
expresamente el ámbito de control de la Corte- en la que el alto tribunal se arroga la competencia para revisar el contenido de la modificación y decide, en cada caso, si se trata de una reforma o de una sustitución.

\section{CONCLUSIONES}

Se pueden concluir que, en primer lugar, la Corte Constitucional incurrió en una mutación constitucional cuando se arrogó la competencia para verificar que la modificación a la $\mathrm{CN}$ fuera una sustitución y no una reforma.

En segundo lugar, si la teoría de los límites competenciales al poder de reforma es una teoría inacabada, salvo que el propio tribunal asuma una posición de auto restricción judicial, hoy cuenta con patente de corso para determinar lo que es reforma y lo que es sustitución, según que el contenido de la modificación a la $\mathrm{CN}$ le parezca inconveniente o políticamente inaceptable.

En tercer lugar, la posibilidad de que la Corte Constitucional revise los vicios de competencia en el sentido que lo viene haciendo, es una clara muestra de la debilidad de la democracia en Colombia, la cual no se expresa a través ni del Congreso ni del referendo, sino que canaliza sus necesidades a través de un órgano de naturaleza contra mayoritaria carente de todo control judicial y político.

Por último y cuarto lugar, la teoría de los límites constitucionales es una expresión del neoconstitucionalismo predominante hoy en Colombia, y que se traduce no solo en una penetración del texto constitucional y de la opinión de la Corte Constitucional en todos los ámbitos de la vida nacional, sino también en el desbordamiento del concepto mismo de constitución, el que deja hoy pocos espacios para el principio democrático.

\section{BIBLIOGRAFÍA}

Bernal Cuéllar, J. y Montealegre Lynett, E. (1995). El Proceso Penal. Bogotá: Universidad Externado de Colombia.

Cajas Sarria, M. (2007). Acerca del control judicial dela reforma constitucional en Colombia. Revista Iberoamericana de Derecho Procesal Constitucional, 7, 19-40. Disponible en: https://www.researchgate.net/profile/Mario_Cajas/publication/28249343_Acerca _del_control_judicial_de_la_reforma_constitucional_en_Colombia/links/0deec5271c8b173d54000000.pdf.

Chinchilla Herrera, T. E. (2010). Concepciones sobre el Juez Constitucional en la Reforma de 1910: una cuestión de confianza. Diálogos de Derecho y Politica, 1 (3). Disponible en: http://aprendeenlinea.udea.edu.co/revistas/index.php/derypol/article/ view/5121. 
Cruz Villalón, P. (1987). La formación del sistema europeo de control de constitucionalidad. Madrid: CECM.

De Vergottini, G. (1985). Derecho Constitucional Comparado. Madrid: Espasa-Calpe.

Dworkin, R. (1984). Los derechos en serio. Barcelona: Ariel.

Esguerra Portocarrero, J. C. (2004). La protección constitucional del ciudadano. Bogotá: Legis.

Favoreu, L. (1994). Los tribunales constitucionales. Barcelona: Ariel.

Ferreres Comella, V. (2011). Una defensa del modelo europeo de control de constitucionalidad. Madrid: Marcial Pons.

García-Atance, M. (2002). Reforma y permanencia constitucional. Madrid: CEPC.

García de Enterría, E. (1984). La Constitución como norma y el Tribunal Constitucional. Madrid: Cívitas.

García de Vega, P. (1985). La reforma constitucional y la problemática del poder constituyente. Madrid: Tecnos.

Hart, H. L. (2004). El concepto del derecho. Buenos Aires: Abeledo-Perrot.

Loewenstein, K. (1970). Teoría de la Constitución. Barcelona: Ariel.

López Medina, D. (2009). El derecho de los jueces. Bogotá: Legis.

Moreno Millán, F. (2007). La acción pública de inconstitucionalidad, efectos temporales de sus fallos. Bogotá: Leyer.

- (2013). La acción ciudadana de inconstitucionalidad. Cali: Universidad de San Buenaventura Cali.

Ramírez Cleves, G. (2005). Limites de la reforma constitucional en Colombia. Bogotá: Universidad Externado de Colombia.

- (2006). El control material de las reformas constitucionales mediante acto legislativo a partir de la jurisprudencia establecida en la sentencia C-551 de 2003. Revista Derecho del Estado, 18, 3.

Ver publicación compartida

Restrepo Piedrahita, C. (2003). Constitucionales Politicas Nacionales de Colombia. Bogotá: Universidad Externado de Colombia.

Rodríguez, C. (1997). La decisión judicial, el debate Hart-Dworkin. Bogotá: Siglo del hombre, Universidad de Los Andes.

Sánchez Ferriz, R. (1993). Introducción al Estado constitucional. Barcelona: Ariel.

Vigo, R. (2006). Interpretación jurídica. Buenos Aires: Rubinzal-Culzoni.

Zagrebelsky, G. (1977). La giustizia Costituzionale. Turín: Il Mulino.

\section{JURISPRUDENCIA}

Corte Constitucional de Colombia. SC-544, 1992.

Corte Constitucional de Colombia. SC-350, 1994.

Corte Constitucional de Colombia. SC-085, 1995.

Corte Constitucional de Colombia. SC-685, 1996.

Corte Constitucional de Colombia. SC-543, 1998.

Corte Constitucional de Colombia. SC-582, 1999. 
Corte Constitucional de Colombia. SU-047, 1999.

Corte Constitucional de Colombia. SC-1373, 2000.

Corte Constitucional de Colombia. SC-1404, 2000.

Corte Constitucional de Colombia. SC-737, 2001.

Corte Constitucional de Colombia. SC-992, 2001.

Corte Constitucional de Colombia. SC-487, 2002.

Corte Constitucional de Colombia. SC-614, 2002.

Corte Constitucional de Colombia. SC-551, 2003.

Corte Constitucional de Colombia. SC-1200, 2003.

Corte Constitucional de Colombia. SC-668, 2004.

Corte Constitucional de Colombia. SC-757, 2004.

Corte Constitucional de Colombia. SC-971, 2004.

Corte Constitucional de Colombia. SC-1124, 2004.

Corte Constitucional de Colombia. SC-1040, 2005.

Corte Constitucional de Colombia. SC-1040, 2005.

Corte Constitucional de Colombia. ST-025, 2005.

Corte Constitucional de Colombia. SC-472, 2006.

Corte Constitucional de Colombia. SC-720, 2006.

Corte Constitucional de Colombia. SC-757, 2008.

Corte Constitucional de Colombia. SC-228, 2009.

Corte Constitucional de Colombia. SC-040, 2010.

Corte Constitucional de Colombia. Auto 281, 2010.

Corte Constitucional de Colombia. SC-577, 2011.

Corte Constitucional de Colombia. SC-1017, 2012.

Corte Constitucional de Colombia. SC-258, 2013.

\section{LEGISLACIÓN}

Acto Legislativo 2 de 1977.

Acto Legislativo 1 de 1979.

Acto Legislativo 3 de 2002.

Acto Legislativo 1 de 2003.

Acto Legislativo 2 de 2003.

Acto Legislativo 2 de 2004.

Acto Legislativo 1 de 2005.

Acto Legislativo 1 de 2007.

Acto Legislativo 1 de 2008.

Acto Legislativo 1 de 2009.

Acto Legislativo 1 de 2011.

Acto Legislativo 2 de 2011.

Acto Legislativo 4 de 2011.

Acto Legislativo 5 de 2011.

Acto Legislativo 6 de 2011. 
Constitución Política Nacional de Colombia de 1821.

Constitución Política Nacional de Colombia de 1886.

Constitución Política Nacional de Colombia de 1991.

Ley 5 de 1992, Colombia, por la cual se expide el Reglamento del Congreso; el Senado y la Cámara de Representantes.

Ley 134 de 1994, Colombia, por la cual se dictan normas sobre mecanismos de participación ciudadana.

Ley 796 de 2003, Colombia, por la cual se convoca un referendo y se somete a consideración del pueblo un proyecto de Reforma Constitucional.

Ley 1327 de 2009, Colombia, por la cual se convoca a un referendo constitucional y se somete a consideración del pueblo un proyecto de Reforma Constitucional. 